\title{
A universal outcome measure for headache treatments, care-delivery systems and economic analysis
}

Timothy J Steiner ${ }^{1,2 *}$, Mattias Linde ${ }^{1,3,4}$ and Petra Schnell-Inderst ${ }^{5}$

\begin{abstract}
Background: The first manuscript in this series delineated a model of structured headache services, potentially cost-effective but requiring formal cost-effectiveness analysis (CEA). We envisaged a need for a new outcome measure for this purpose, applicable to all forms of treatment, care and care-delivery systems as opposed to comparisons of single-modality treatments.

Conception and delineation: A literature review confirmed the lack of any suitable established measure. We prioritised construct validity, simplicity, comprehensiveness and expression in intuitive units. We noted that pain was the key burdensome symptom of migraine and episodic tension-type headache (TTH), that pain above a certain level was disabling, that it was difficult to put economic value to pain but relatively easy to do this for time, a casualty of headache leading to lost productivity. Alleviation of pain to a non-disabling level would be expected to bring restoration of function. We therefore based the measure on time spent in the ictal state (TIS) of migraine or TTH, either as total TIS or proportion of all time. We expressed impact on health, in units of time, as TIS*DW, where DW was the disability weight for the ictal state supplied by the Global Burden of Disease (GBD) studies. If the time unit was hours, TIS*DW yielded hours lived with (or lost to) disability (HLDs), in analogy with GBD's years lived with disability (YLDs).
\end{abstract}

Utility assessment: Acute treatments would reduce TIS by shortening attack duration, preventative treatments by reducing attack frequency; health-care systems such as structured headache services would have these effects by delivering these treatments. These benefits were all measurable as HLDs-averted. Population-level estimates would be derived by factoring in prevalence, but also taking treatment coverage and adherence into account. For healthcare systems, additional gains from provider-training (promoting adherence to guidelines and, therefore, enhancing coverage) and consumer-education (improving adherence to care plans), increasing numbers within populations gaining the benefits of treatments, would be measurable by the same metric.

Conclusions: The new outcome measure expressed in intuitive units of time is applicable to treatments of all modalities and to system-level interventions for multiple headache types, with utility for CEA and for informing health policy.

\footnotetext{
* Correspondence: t.steiner@imperial.ac.uk

${ }^{1}$ Department of Neuromedicine and Movement Science, NTNU Norwegian

University of Science and Technology, Edvard Griegs gate, Trondheim,

Norway

${ }^{2}$ Division of Brain Sciences, Imperial College London, London, UK

Full list of author information is available at the end of the article
}

(c) The Author(s). 2021 Open Access This article is licensed under a Creative Commons Attribution 4.0 International License, which permits use, sharing, adaptation, distribution and reproduction in any medium or format, as long as you give appropriate credit to the original author(s) and the source, provide a link to the Creative Commons licence, and indicate if changes were made. The images or other third party material in this article are included in the article's Creative Commons licence, unless indicated otherwise in a credit line to the material. If material is not included in the article's Creative Commons licence and your intended use is not permitted by statutory regulation or exceeds the permitted use, you will need to obtain permission directly from the copyright holder. To view a copy of this licence, visit http://creativecommons.org/licenses/by/4.0/. The Creative Commons Public Domain Dedication waiver (http://creativecommons.org/publicdomain/zero/1.0/) applies to the data made available in this article, unless otherwise stated in a credit line to the data. 
Keywords: Headache disorders, Structured headache services, Cost-effectiveness analysis, Health technology assessment, Outcome measure, Health policy, Global Campaign against Headache

\section{Introduction}

The first manuscript in this themed series noted the high ill-health, disability and economic burdens arising from headache disorders worldwide, which persist despite the existence of effective treatments [1]. It put the blame on widespread failures of health services to deliver these treatments to those who might benefit, failures attributable to lack of awareness, lack of political will and lack of an evidence-based solution [1]. The authors addressed all three of these as barriers to be surmounted, ultimately proposing a model of structured headache services as the health-care solution [1]. They predicted, with some evidential support $[2,3]$, that this model would be cost-effective and might, potentially, be cost saving if appropriately implemented, but they recognised that these predictions required testing in formal costeffectiveness analysis (CEA) [1].

Here lay a difficulty: on what outcome measure(s) might economic evaluation of headache services, as opposed to single-modality treatments such as acute or preventative drugs, be based? Previous economic evaluations have used both disease-specific clinical outcomes and generic measures [4-11], but choice among the former has been restricted almost exclusively to those used and reported in randomised clinical trials (RCTs), which are rarely designed to support economic evaluation.

Our purposes in this manuscript were first to review the literature for candidate measures in common use that might serve the requirements of economic evaluation; second, subject to findings, to develop a new measure applicable equally to all forms of treatment, care and care-delivery systems for headache regardless of type; and third to demonstrate its broad utility in these applications.

\section{Review of potential candidate measures}

\section{Literature search}

We did not review the literature systematically for all outcome measures applied to headache care: rather, we judged that our purpose would be better served by a more focused review. We limited it to efficacy measures previously employed in assessing treatments of migraine and/or tension-type headache (TTH), these being the principal headache disorders for which headache services cater [1]. Our envisaged setting was a world in which large numbers of people are affected by headache but have limited treatment options because they live either in developing countries where health-care resources are generally limited or in developed countries where health care for headache has low priority $[1,2,12]$. Therefore, we further limited the review to treatment that would not require consultation with a physician. With this in mind, we looked specifically at RCTs of acetylsalicylic acid (ASA), a choice driven by several considerations. ASA is a first-line treatment with proven efficacy for both migraine and TTH [13-17], it is listed by the World Health Organization (WHO) as an essential medicine for treatment of acute migraine [18], and it is readily available at low cost worldwide.

Also in mind was that only measures expressible in intuitive units, to which economic value could be assigned, would be suitable for purpose. This ruled out a range of multifactorial patient-reported outcome measures (PROMs) [19-25] including the Headache Impact Test (HIT-6) [20] and Headache Under-Response to Treatment (HURT) questionnaire [25], generic [26-28] and disease-specific quality-of-life measures $[29,30]$ and measures based on WHO's Classification of Functioning, Disability and Health (ICF) [31-33]. It did not rule out measures based on the Disability-Adjusted Life Year (DALY) since this was developed expressly for CEA and to inform health policy and priority-based decision making [3].

Finally, we limited the review to a 20 -year period from 1988, when the International Classification of Headache Disorders (ICHD) first introduced operational and widely accepted diagnostic criteria for migraine and TTH [34]. This period saw the development and introduction of triptans, and drug-company sponsorship not only of RCTs in which ASA was a comparator but also of RCTs comparing ASA with placebo. By the end of this period, this activity had effectively ceased.

The search included MEDLINE and EMBASE along with the Cochrane Library. We supplemented it by reference to recommendations in the two sets of clinical trials guideline published by the International Headache Society (IHS), in migraine [35] and TTH [36].

It found ten studies describing efficacy measures, seven in migraine [37-43] and three in TTH [44-46]. All recruited from outpatient settings and most (all but one of the TTH studies [45]) included patients with headache at base line of at least moderate intensity.

\section{Commentary}

Headache relief after $2 \mathrm{~h}$ (HR2) was the preferred endpoint in migraine trials. It was used as the primary outcome measure in five $[37,39,41-43]$ and secondary in 
one [38], and defined in all in accordance with IHS clinical trials guidelines [35] as reduction in headache pain from moderate or severe at base line to mild or no pain at $2 \mathrm{~h}$. Sustained headache relief over $24 \mathrm{~h}$ (SHR24) was defined, also in accordance with IHS guidelines [35], as HR2 without, then, use of rescue medication or headache recurrence within $24 \mathrm{~h}$. Five trials reported SHR24 or allowed its calculation by reporting HR2, recurrence rate and use of rescue medication [38, 39, 41-43]. Six trials reported pain-freedom (no pain) at $2 \mathrm{~h}$ (PF2) as a secondary endpoint, either directly or by inference from visual analogue scale (VAS) measurements [38-43]. Less frequently used were sustained pain-freedom over $24 \mathrm{~h}$ (SPF24), pain intensity difference (PID) calculated as pain intensity on a VAS at base line minus pain intensity at various later times, the sum of PIDs (SPID) recorded at multiple time points and weighted according to the time between observations, and functional impairment (disability) assessed subjectively.

All three TTH studies [44-46] used pain relief, as a change from base line, as the primary endpoint, but defined it differently: at different times and by different means, either on a verbal rating scale (VRS) or on a VAS, sometimes expressed relatively rather than in absolute terms. All studies assessed PID at intervals after treatment, but over different total time periods. Less frequently reported were SPID (although one TTH study used SPID over $4 \mathrm{~h}$ as the primary endpoint [44]), use of rescue medication and functional impairment over $24 \mathrm{~h}$.

Of these measures, HR2 has obvious utility. In the migraine trials it was achieved by about half of ASA users, in line with the general perception that a similar proportion with migraine in the general population could effectively self-manage [1] (although people recruited from outpatient settings might not be representative of these). HR2 also distinguished clearly between active and placebo treatments in the trials. However, its 2-hour timeframe in the context of episodes lasting 4-72 $\mathrm{h}$ untreated [34] is too short to express efficacy fully. In $\mathrm{TTH}$, pain relief at $2 \mathrm{~h}$ (PR2) may be analogous to HR2, but with a limitation (discussed later): it cannot be universally applied, being observable only in those with at least moderate pain at base line while pain in TTH may, often, be only mild [34]. PR2 was achieved in the two relevant TTH trials by about three quarters of ASA users [44, 46], with, again, clear distinction between active and placebo treatments.

Pain-freedom may be the outcome most desired by people treating either disorder, and the IHS guidelines for clinical trials in migraine recommend PF2 as primary efficacy endpoint [35]. But PF2 in the migraine trials we reviewed was uninformative about treatment effect in more than three quarters of ASA users because they did not achieve it, although many of these might nonetheless have had worthwhile benefit [47]. Again, it covers too short a timeframe to express efficacy fully. The same endpoint, reported as the conceptually approximate "total" PR2 in the TTH trials we reviewed, is recommended as primary efficacy endpoint by the IHS guidelines for clinical trials in TTH [36], but remains similarly limited.

PID equates reductions in pain intensity (whether absolute or proportional) regardless of its initial level. Its inbuilt assumptions about the pain intensity continuum, and that perceived change is independent of base line, render it conceptually dubious. Thus, for example, on a VAS or NRS, PID attaches similar meaning to absolute reductions from 10 to 7 (remaining severe), from 5 to 2 (moderate becoming mild) and from 3 to 0 (pain resolution). Expressed proportionately (often as reduction by $50 \%$ ), it gives equal weight to reductions from 8 to 4 and from 4 to 2 . Further, when PID measurements are made serially, each is not independent of the one before. PID therefore has validity only at a single pre-specified time point. SPID, an integrating measure over time, circumvents the last problem but is highly cumbersome, requiring multiple timed measurements if applied over a timeframe long enough to express efficacy adequately.

While use of rescue medication is a measure reflecting primary inefficacy, and expresses an important dimension of outcome, it is applicable only to subsets experiencing this: in the trials, about $60 \%$ taking ASA for migraine and $85 \%$ for TTH did not require rescue. Conversely, while headache recurrence is also an important outcome for those experiencing it, recurrence within $24 \mathrm{~h}$ as an endpoint is possible only in those who initially respond. SHR24, in either migraine or TTH (and defined in the same way in both), takes account of both recurrence and use of rescue medication while also capturing primary efficacy. It is measurable in everybody with at least moderate base-line pain (including all randomised patients in most RCTs - an important consideration) by observations at two time points only. While it represents an imperfect outcome [47], several economic studies have used it as the option that best reflected treatment success $[4,7,8]$.

None of these disease-specific outcome measures embrace disability. Both sets of IHS guidelines recommend assessing disability by VRS as secondary outcome measures $[35,36]$. For most people, the immediate objective of treating acute episodes of migraine or TTH, the two causes of most of the headache-attributed ill-health burden [48-52], is to relieve symptoms, of which headache is usually predominant [15]. Preventative drugs have the same but more distanced objective, relieving symptom burden by reducing future episode frequency [15]. In either case, the purpose beyond alleviating pain is to minimise disability, a key consequence of pain [53]: acute 
treatment is taken in the hope of restoring normal function as quickly as possible, preventative treatment to avert its loss. This suggests that disability should be the focus of outcome measurement [53, 54], particularly since disability is the presumed cause of lost productivity, the major contributor to economic cost [55].

It should be noted that this presumption is not a given [56]. At issue are the meaning of disability, to which we make reference later, and the relationship between disability (however defined) and lost productivity, which is complex [57]. Nevertheless, a disability-based outcome measure could serve all purposes: comparative evaluation of headache treatments of all types, effectiveness assessment of care-delivery systems, and economic analysis. Of the trials, only a minority reported functional impairment $[40,42,43,46]$, always as a subjective assessment: in migraine, on either a 4-point ("none", "mild", "moderate" or "severe") [43] or a 5-point VRS ("able to perform all activities", "daily activities require a little additional effort", "daily activities require some additional effort", "daily activities require a great deal of additional effort" or "unable to perform daily activities") [42]. These, applied retrospectively to the attack as a whole, were unsophisticated assessments; lacking any time dimension, they could not be useful in comparisons of treatments of different modalities or in the context of economic analysis.

\section{Conclusions}

In short, no outcome measure in common use could meet all requirements. More specifically, to our earlier question ("On what outcome measure(s) might economic evaluation of headache services, as opposed to single-modality treatments such as acute or preventative drugs, be based?"), asked in an envisaged but real world in which many people are affected by headache but have limited treatment options [1], none of these is the answer.

\section{Development of a new measure Conception and delineation}

We prioritised four properties of a new measure: construct validity, of course, but also simplicity, comprehensiveness and expression in intuitive units. We also took the view that a universal outcome measure must in some manner take account of clinically important adverse events (AEs) reasonably attributable to the intervention.

As a starting point, with regard to construct validity, we noted that pain was the key burdensome symptom of both migraine and episodic TTH, and that pain above a certain level was disabling $[52,53]$. We recognised also that endpoints expressing key symptoms were not always amenable to economic analysis: it was difficult, for example, to put an economic value to pain. On the other hand, it would be relatively easy to do this for time, very often a casualty of headache leading to the reportedly high indirect costs of lost or reduced productivity [55, 58, 59]. Alleviation of pain to a non-disabling level would be expected to bring restoration of function; the sooner this was achieved, the greater the recovery of useful (productive) time that would otherwise be lost.

With regard to comprehensiveness, we also noted that, to make comparisons across diseases, generic measures of health outcomes were needed. The DALY, used by WHO and the Global Burden of Disease (GBD) study [1, 48-51]), is a generic summary measure of lost health, having utility in relative assessments of health benefits gained by interventions. DALYs are the sum of years of life lost to premature mortality (YLLs) and years lost to disability (YLDs); because headache does not reduce life expectancy, DALYs here equate to YLDs. GBD2013 derived disability weights (DWs: valuations on a $0-1$ scale, where 1 = full health) for health states consequent upon diseases (including the ictal states of migraine and TTH) [60] from a worldwide survey eliciting comparative judgements about these health states. YLDs attributable to health states are calculated as the product of time in the health state and its DW. While this became the basis for estimating global burdens attributable to each disease, and ranking diseases accordingly [48-51], it also, along with SHR24, provided us with the foundations on which to develop a universal outcome measure for headache.

With reference to earlier comments, we say more later about the meaning of disability in the specific context of YLDs and DWs (see Discussion). In the following, we use the term with this meaning.

\section{Hours lost to (or lived with) disability (HLDs)}

We based the measure on time spent in the ictal state (TIS) of either migraine or TTH, expressed as total time (tTIS) or proportion of total time (pTIS). In the context of RCTs looking at single acute episodes, TIS was expressed simply by attack duration with or without treatment. In the more general contexts of individual and population health, tTIS and pTIS were expressed for a defined period of time in the former case as the product of mean duration and mean attack frequency and in the latter case as $\mathrm{D}^{*} \mathrm{~F}^{* *}$ prevalence, $\mathrm{D}$ and $\mathrm{F}$ here representing the population means of attack duration and frequency. For each disorder (migraine or TTH), the absolute impact on health was expressed, in units of time, as tTIS*DW during a prescribed period and the relative impact, as a proportion, as pTIS*DW. If the time unit was hours, the most logical and practical, tTIS*DW yielded hours lost to disability (HLDs), in analogy with YLDs. 


\section{HLDs averted}

Acute treatments would diminish (avert) HLDs by shortening attack duration, preventative treatments by reducing attack frequency; health-care systems such as structured headache services [1] would have these effects or would supplement them by delivering or enhancing delivery of these treatments. These benefits would all be measurable as HLDs averted.

\section{Utility assessment}

To demonstrate the measure's broad utility, we theoretically modelled its application in acute and preventative therapies, and in the evaluation of a service-delivery model such as structured headache services [1] providing migraine care. In each case, we modelled the effects of the intervention on either attack duration or attack frequency, at individual or population levels.

Figure 1 is a figurative depiction of how the outcome measure applies to acute therapy for migraine in an individual. On the left is the imagined course of an untreated attack of 14 hours' duration. From onset, pain would increase to its maximum over several hours, plateau, then decline rapidly as the attack ended spontaneously. For the period of $13 \mathrm{~h}$ during which pain was greater than mild ( $>1$ on the scale $0-3$ ), disability (depicted below on the left) would be according to the DW for the ictal state from GBD2013 [60] (i.e., 44\%). On the right, acute therapy taken at $1 \mathrm{~h}$ and achieving efficacy (i.e., SHR24) would reduce pain to mild within two hours, without recurrence. Disability, again modelled below, would be $44 \%$ until pain was reduced and then zero according to the assumption that mild pain was not disabling $[52,53]$. Thus, duration would be reduced from 14 to $3 \mathrm{~h}$, with 11 HLDs averted (bottom right)

Population estimates would involve interpreting SHR24 reports from RCTs (for example [17, 61, 62]): acute drugs would reduce pain intensity from disabling levels to non-disabling within $2 \mathrm{~h}$, without recurrence, in the proportion of attacks stipulated by SHR24 as a reported outcome measure. Thus, if treatment was taken at onset, tTIS would effectively be reduced in this proportion of attacks from expected duration to $2 \mathrm{~h}$, where expected duration might be the mean placebo-treated duration in an RCT or the mean derived from population-based data. An imaginary numerical example for a population is given in Table 1 .

For preventative treatments, tTIS would be reduced directly in proportion to effect on attack frequency. Table 2 is an imaginary numerical example for an individual, for whom untreated $(\mathrm{uF})$ and treated $(\mathrm{tF})$ attack frequencies were observable; at population level it would be necessary to factor in estimates of treatment effect $(\mathrm{dF})$ and its probability $(\mathrm{pE})$ derived from RCTs.

All of these effects would ultimately be expressed in HLDs averted by introduction of the factor DW [60]. For acute treatments, in the context of a single attack, this is illustrated in Fig. 1. For multiple attacks over
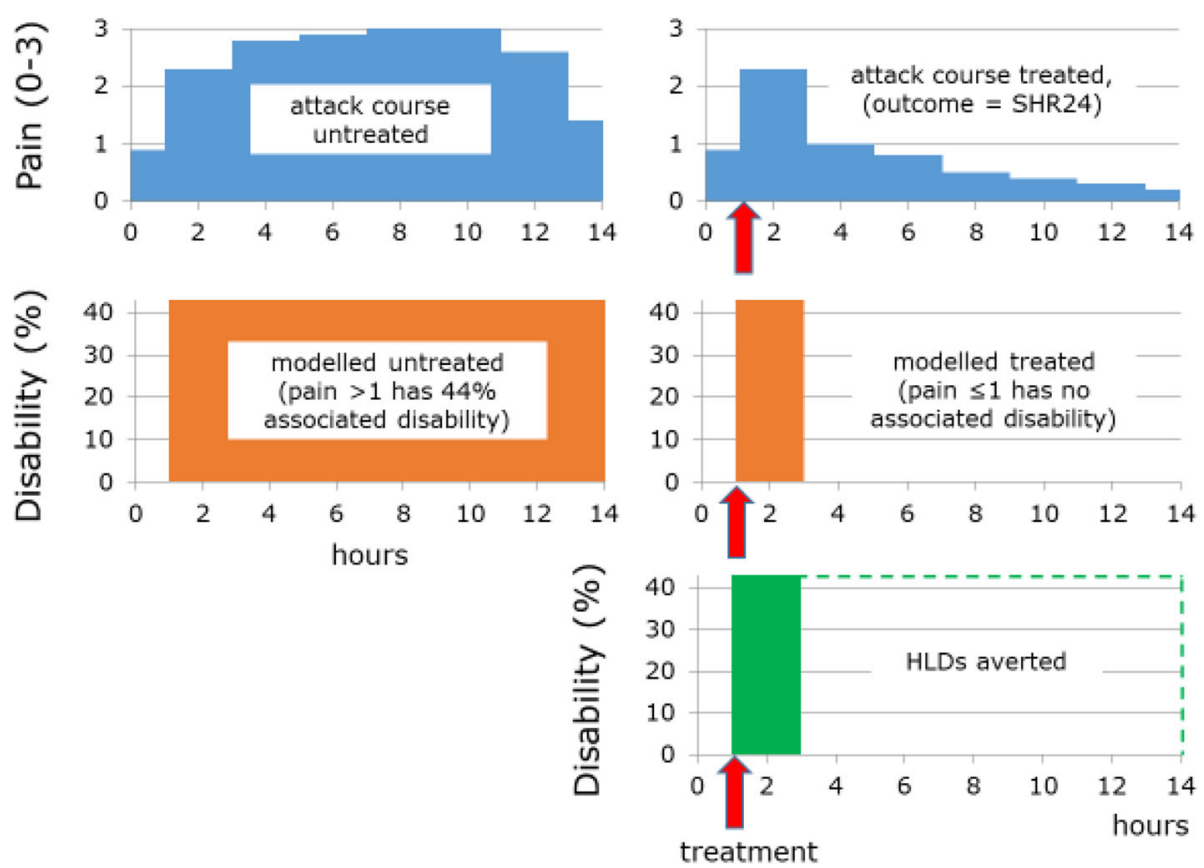

Fig. 1 Figurative depiction of how the outcome measure applies to acute therapy over the course of a single migraine attack in an individual (see text for explanation) 
Table 1 Imaginary numerical example of acute treatment effect in a population

\begin{tabular}{ll}
\hline Mean untreated attack duration (uD) & $12 \mathrm{~h}$ \\
Successfully-treated attack duration (tD) & $2 \mathrm{~h}$ \\
Change in mean time in ictal state (dTIS) & $\mathrm{uD}-\mathrm{tD}=12-2=10 \mathrm{~h}$ \\
Number of attacks treated (nA) & 100 \\
Probability of treatment success (SHR24) (pE) & $50 \%$ \\
Change in total time in ictal state (dtTIS) & $\mathrm{dTIS}^{*} \mathrm{nA}^{*} \mathrm{pE}=10^{*} 100^{*} 50 \%=500 \mathrm{~h}$ \\
\hline
\end{tabular}

time, HLDs averted would be dtTIS*DW, with the assumption that treatment was taken before or as soon as headache became disabling so that there was no measurable health loss before treatment. The proportion $\mathrm{pE}$ deemed to be successfully treated could be reducible to take account of clinically important AEs according to their frequencies by negating success when they occurred. (This adjustment might be over-conservative, but is an option available according to purpose. It would not satisfactorily account for serious AEs, which always require separate recording.) For preventative treatments, effects would be expressed in HLDs averted through the same product dtTIS*DW, again reducible, should the purpose require it, by the proportion of treatment discontinuations due to AEs.

Should both acute and preventative drugs be taken, interaction would be duly taken account of by projecting the potential effect of the former alone onto attacks not averted by the latter.

Population-level estimates would be derived by factoring in prevalence, but also taking treatment coverage and adherence into account as well as efficacy. For health-care-system interventions, effects should be expressed through gains in these (more people receiving and taking appropriate treatments) as the consequences of provider-training (enhancing care coverage) and consumer-education (improving adherence) [1], each by specifiable amounts. Improvements in either would proportionately increase numbers within populations gaining the benefits of treatments, and therefore the total population-level benefits measurable by the same metric.

For TTH, the measure is similarly applied, but with the limitation arising from the fact that TTH may only be mild [34] - more commonly so than migraine. Because the DW for the ictal state of TTH is much lower

Table 2 Imaginary numerical example of preventative treatment effect in an individual

\begin{tabular}{ll}
\hline Untreated attack frequency (uF) & $60 /$ year \\
Treated attack frequency (tF) & $30 /$ year \\
Change in attack frequency (dF) & $30 /$ year \\
Attack duration (D) & $14 \mathrm{~h}$ \\
Change in total time in ictal state (dtTIS) & $\mathrm{dF}^{*} \mathrm{D}=30^{*} 14=420 \mathrm{~h} /$ year \\
\hline
\end{tabular}

(0.037 as opposed to 0.441 for migraine [60]), HLDs are far fewer. These limitations are discussed below.

\section{Discussion}

This presentation has described the conception and delineation of a new universal outcome measure applicable to treatments of all modalities of headache of multiple types (migraine or TTH, but also of other types manifesting as attacks definable in terms of duration and frequency) and expressed in intuitive units of time. Its development relied on disability as estimated in the GBD studies [48-51]. The measure was applied in theoretical scenarios to both acute and preventative migraine treatments, to individuals and populations, and to systems designed to deliver these treatments, with all outcomes expressed by the same single metric rooted in the YLD.

What was the need? Multiple outcome measures existed already for treatments of migraine and TTH, and several were widely accepted, though not, perhaps, with universal agreement [47, 63]. These performed the important clinical function of allowing comparisons of treatments to inform choice. Where they fell short was in comparisons of treatments of different modalities. In the context of economic analysis, with the purpose of valuing interventions of different types relative to each other, existing measures were applied with difficulty. When interventions were at the level of service organisation and delivery rather than provision of singlemodality treatments (such as drugs), they were unhelpful. When the purpose was to inform prioritisation within health policy of resource allocation across diseases, they failed altogether. The need we recognised was defined by this last purpose, but all purposes are served by the new measure.

HLDs as the unit of our measure are calculated by introducing DW for the ictal state (of either disorder) as a factor. In the context of interventions only for migraine, our modelling example, DW is a constant (set at 0.441 by GBD2013 [60]). It is not, therefore, essential for comparisons between interventions at whatever level (acute, preventative or care-delivery system). However, when other headache types are also of interest, with different DWs, and certainly when other (non-headache) 
diseases are comparators of interest, DWs are the essential enabling factor, as they are for YLDs [48-51].

However, despite their name, DWs reflect public preferences for health states (and therefore ill health in its broadest sense), not disability [56, 57, 64]. This does raise a question about the construct validity of our measure, which, we argued, was grounded on disability and its effect on productivity as the key elements of headache-attributed burden against which interventions were or should be directed. Whereas time is noted to be a casualty of disabling headache, and, to the extent that time is used productively, this leads to lost productivity, and whereas both absenteeism from work and presenteeism are observed consequences at least of migraine $[55,59]$, there is a pressing need for empirical evidence showing the relationship between YLDs (and HLDs) and lost productivity. Economic analyses seeking to incorporate indirect costs, and especially those doing so to assess the value of headache treatment, require knowledge of the presumed but uncertain relationship between HLDs averted and recovered productivity. Later papers in this themed series go some way to addressing this [56, 57].

HLDs averted are a conservative measure. Several of the assumptions behind its conception and application were conservative. Important among these was that efficacy is all-or-nothing: SHR24 either occurs or it does not. Basing HLDs averted on SHR24 led to conservative effect-estimation in two ways: lesser but possibly still worthwhile effects would not be counted, and no higher value would be attached to greater effects such as sustained pain-freedom. This is justifiable in CEA (conservatism rules in economic analysis), and we would argue that it is not problematic in clinical contexts, where serial assessments of patients are made relatively to assess change. In GBD evaluations, disability is also all-ornothing: it is at the level stipulated by the DW for the health state (44.1\% for the ictal state of migraine [60]) or it is zero. More contentious is our suggestion that attacks otherwise successfully treated (positive SHR24) but attended by clinically important AEs (but not serious AEs) might optionally be discounted. This may be justified in economic analysis, but clinically appears overconservative: a more nuanced approach may be more appropriate. Of course, no benefit can be attributed to preventative treatments necessarily discontinued because of AEs.

We should note that among the limitations of this presentation are other assumptions. One, that mild headache is not associated with disability, is a standard assumption $[52,53]$ not needing justification. A second, that acute treatment is initiated before or as soon as pain becomes disabling, is an assumption necessary to establish a time zero for purposes of effect calculation. It reflects recommended practice [13-15], to be promoted by public education as part of structured headache services [1], and perhaps, therefore, also requires no justification.

Specific limitations, identified earlier, apply to TTH. Because it discounts mild pain, the measure has a more restricted application to TTH than to migraine: TTH is, usually but not always, a mild-to-moderate headache [34]. When base-line pain is mild, the assumption of no disability attributable to it accords zero value to it. In the present context this is of little consequence since mild TTH does not call upon structured headache services [1], or, usually, professional intervention of any sort.

The DW for the ictal state of TTH is much lower than that of migraine: 0.037 as opposed to 0.441 [60]. Of course, this valuation reflects the very fact that TTH is commonly mild. The consequences are that attributable HLDs are far fewer and health care can achieve relatively little in terms of HLDs averted. In other words, the measure is insensitive to TTH. The same is true of estimates of YLDs attributed to TTH in the GBD studies [48-51], but it is a consequence of the public perception of TTH on which its DW is based [60].

\section{Conclusions}

We have described the development of a new universal outcome measure expressed in intuitive units of time and applicable to treatments of all modalities of headache of multiple types. The measure equips economic analysis of interventions, including implementation of structured headache services, for purposes including informing health policy.

\section{Abbreviations}

AE: Adverse event; ASA: Acetylsalicylic acid; CEA: Cost-effectiveness analysis; DALY: Disability-adjusted life year; $\mathrm{dF}$ : Change in frequency (of attacks); dTIS: Change in time in ictal state; dtTIS: Change in total time in ictal state; DW: Disability weight; GBD: Global burden of disease (study); HLD: Hour lost to (or lived with) disability; HR: Headache relief; IHS: International Headache Society; $\mathrm{MOH}$ : Medication-overuse headache; nA: Number of attacks treated; PF: Pain-freedom; PID: Pain intensity difference; $\mathrm{pE}$ : Probability of treatment success; PROM: Patient-reported outcome measure; RCT: Randomised

controlled trial; SHR: Sustained headache relief; SPF: Sustained pain-freedom; SPID: Sum of pain intensity differences; tD: Duration of treated attack; tF: Treated attack frequency; TIS: Time in ictal state; TTH: Tension-type headache; tTIS: Total time in ictal state; uD: Duration of untreated attack; UF: Untreated attack frequency; UK: United Kingdom; VAS: Visual analogue scale; VRS: Verbal rating scale; WHO: World Health Organization; YLD: Year lived with disability; YLL: Year of life lost

\section{Acknowledgements}

We are grateful to Dr Dan Chisholm of World Health Organization for guidance in the methodology of CEA applied to structured headache services and the application to it of the outcome measure, and for advice in structuring this manuscript.

\section{Authors' contributions}

TJS and PS-I perceived the need for the new measure, especially to support economic analyses, and conceived and delineated it. ML and TJS worked on its application to health-care delivery systems and CEA. TJS and PS-I drafted 
parts of the manuscript, which TJS assimilated. All authors contributed to manuscript revision and approved the final version.

\section{Funding}

Bayer HealthCare supported meetings in Munich between PS-I and TJS to conceive and delineate the measure, without any influence on these processes or benefit in return. Lifting The Burden supported meetings between $M L$ and TJS.

\section{Availability of data and materials}

Not applicable.

\section{Declarations}

\section{Ethics approval and consent to participate}

Not applicable.

\section{Consent for publication}

Not applicable.

\section{Competing interests}

TJS is a Director and Trustee of Lifting The Burden, a UK-registered nongovernmental organization conducting the Global Campaign against Headache in official relations with the World Health Organization (WHO). Otherwise, no author declares a conflict of interest in relation to the content of this manuscript.

\section{Author details}

${ }^{1}$ Department of Neuromedicine and Movement Science, NTNU Norwegian University of Science and Technology, Edvard Griegs gate, Trondheim, Norway. ${ }^{2}$ Division of Brain Sciences, Imperial College London, London, UK. ${ }^{3}$ Norwegian Advisory Unit on Headache, Department of Neurology and Clinical Neurophysiology, St Olavs University Hospital, Trondheim, Norway. ${ }^{4}$ Tjörn Headache Clinic, Rönnäng, Sweden. ${ }^{5}$ Institute of Public Health, Medical Decision Making and Health Technology Assessment, Department of Public Health, Health Services Research and Health Technology Assessment, Medical Informatics and Technology, UMIT - University for Health Sciences, Hall in Tirol, Austria.

Received: 2 April 2021 Accepted: 26 May 2021

Published online: 01 July 2021

\section{References}

1. Steiner TJ, Jensen R, Katsarava Z, Stovner LJ, Uluduz D, Adarmouch L, Al Jumah M, Al Khathaami AM, Ashina M, Braschinsky M, Broner S, Eliasson JH, Gil-Gouveia R, Gómez-Galván JB, Guðmundsson LS, Herekar AA, Kawatu N, Kissani N, Kulkarni GB, Lebedeva ER, Leonardi M, Linde M, Luvsannorov O, Maiga Y, Milanov I, Mitsikostas DD, Musayev T, Olesen J, Osipova V, Paemeleire K, Peres MFP, Quispe G, Rao GN, Risal A, Ruiz de la Torre E, Saylor D, Togha M, Yu S-Y, Zebenigus M, Zenebe Zewde Y, Zidverc-Trajković J, Tinelli M on behalf of Lifting The Burden: the Global Campaign against Headache (2021) Structured headache services as the solution to the illhealth burden of headache. 1. Rationale and description. J Headache Pain 22 (in press)

2. World Health Organization and Lifting The Burden (2011) Atlas of headache disorders and resources in the world 2011. Geneva: WHO. At: http://www. who.int/mental_health/management/atlas_headache_disorders/en/2011

3. Linde M, Steiner TJ, Chisholm D (2015) Cost-effectiveness analysis of interventions for migraine in four low- and middle-income countries. J Headache Pain 16:15

4. Yu J, Goodman MJ, Oderda GM (2009) Economic evaluation of pharmacotherapy of migraine pain: A review of the literature. J Pain Palliat Care Pharmacother 23:396-408

5. Biddle AK, Shih Y-CT, Kwong WJ (2000) Cost-benefit analysis of sumatriptan tablets versus usual therapy for treatment of migraine. Pharmacotherapy 20 1356-1364

6. Ramsberg J, Henriksson M (2007) The cost-effectiveness of oral triptan therapy in Sweden. Cephalalgia 27:54-62

7. Slof J, Badia X, Magaz S, Láinez MJA, Galván J, Heras J (2005) Cost-efficacy of oral triptans in the treatment of acute migraine. J Med Econ 8:27-43
8. Slof J (2012) Cost-effectiveness analysis of early versus non-early intervention in acute migraine based on evidence from the 'Act when Mild' study. Appl Health Econ Health Policy 10:201-215

9. Witt CM, Reinhold T, Jena S, Brinkhaus B, Willich SN (2008) Costeffectiveness of acupuncture treatment in patients with headache. Cephalalgia 28:334-345

10. National Institute for Health and Care Excellence (2012, updated 2015) Headaches in over 12s: diagnosis and management. Clinical guideline [CG150]. NICE, at: https://www.nice.org.uk/Guidance/CG150. (Surveillance report 2016, at: https://www.nice.org.uk/guidance/cg150/resources/surveilla nce-report-2016-headaches-in-over-12s-diagnosis-and-management-2012nice-guideline-cg150-2721165808/chapter/Surveillance-decision?tab= evidence; Appendix A: Summary of new evidence from surveillance, at: https://www.nice.org.uk/guidance/cg150/evidence/appendix-a-summary-ofnew-evidence-pdf-2721165806)

11. Ruggeri M, Drago C, Rosiello F, Orlando V, Santori C (2020) Economic evaluation of treatments for migraine: an assessment of the generalizability following a systematic review. PharmacoEconomics 38:473-484

12. American Association for the Study of Headache, International Headache Society (1998) Consensus statement on improving migraine management. Headache $38: 736$

13. Diener H-C, Holle-Lee D, Nägel S, Dresler T, Gaul C, Göbel H, Heinze-Kuhn K, Jürgens T, Kropp P, Meyer B, May A, Schulte L, Solbach K, Straube A, Kamm K, Förderreuther S, Gantenbein A, Petersen J, Sandor P, Lampl C (2019) Treatment of migraine attacks and prevention of migraine: Guidelines by the German Migraine and Headache Society and the German Society of Neurology. Clin Translational Neurosci 3:1-40

14. Evers S, Áfra J, Frese A, Goadsby PJ, Linde M, May A, Sándor PS (2009) EFNS guideline on the drug treatment of migraine - revised report of an EFNS task force. Eur J Neurol 16:968-981

15. Steiner TJ, Jensen R, Katsarava Z, Linde M, MacGregor EA, Osipova V, Paemeleire K, Olesen J, Peters M, Martelletti P on behalf of the European Headache Federation and Lifting The Burden: the Global Campaign against Headache (2019) Aids to management of headache disorders in primary care (2nd edition). J Headache Pain 20: 57

16. Kirthi V, Derry S, Moore RA (2013) Aspirin with or without an antiemetic for acute migraine headaches in adults. Cochrane Database Syst Rev 4: CD008041. doi:https://doi.org/10.1002/14651858.CD008041.pub3

17. Derry S, Wiffen PJ, Moore RA (2017) Aspirin for acute treatment of episodic tension-type headache in adults. Cochrane Database Syst Rev 1:CD011888. doi:https://doi.org/10.1002/14651858.CD011888.pub2

18. World Health Organization (2019) The selection and use of essential medicines. WHO, Geneva, p 504

19. Stewart WF, Lipton RB, Simon D, Von Korff M, Liberman J (1998) Reliability of an illness severity measure for headache in a population sample of migraine sufferers. Cephalalgia 18:44-51

20. Kosinski M, Bayliss MS, Bjorner JB, Ware JE Jr, Garber WH, Batenhorst A, Cady R, Dahlöf CG, Dowson A, Tepper S (2003) A six-item short-form survey for measuring headache impact: the HIT-6. Qual Life Research 12:963-974

21. El Hasnaoui A, Vray M, Richard A, Nachit-Ouinekh F, Boureau F: MIGSEV Group (2003) Assessing the severity of migraine: development of the MIGSEV scale. Headache 43: 628-635

22. Cramer JA, Silberstein SD, Winner P (2001) Development and validation of the Headache Needs Assessment (HANA) survey. Headache 41:402-409

23. Jacobson GP, Ramadan NM, Aggarwal SK, Newman CK (1994) The Henry Ford Hospital Disability Inventory (HDI). Neurology 44:837-842

24. Jacobson GP, Ramadan NM, Norris L, Newman CW (1995) Headache disability inventory (HDI): short-term test-retest reliability and spouse perceptions. Headache 35:534-539

25. Steiner TJ, Buse DC, Al Jumah M, Westergaard ML, Jensen $R H$, Reed $M L$, Prilipko L, Mennini FS, Láinez MJA, Ravishankar K, Sakai F, Yu S-Y, Fontebasso M, Al Khathami A, MacGregor EA, Antonaci F, Tassorelli C, Lipton RB on behalf of Lifting The Burden: The Global Campaign against Headache (2018) The headache under-response to treatment (HURT) questionnaire, an outcome measure to guide follow-up in primary care: development, psychometric evaluation and assessment of utility. J Headache Pain 19: 15

26. Ware JE Jr, Sherbourne CD (1992) The MOS 36-item short-form survey (SF36): I. Conceptual framework and item selection. Med Care 30:473-483

27. Ware J Jr, Kosinski M, Keller SD (1996) A 12-item short-form health survey: Construction of scales and preliminary tests of reliability and validity. Med Care 34:220-233 
28. World Health Organization. WHOQOL-BREF. At: https://www.who.int/tools/ whogol/whogol-bref

29. Wagner TH, Patrick DL, Galer BS, Berzon RA (1996) A new instrument to assess the long term quality of life effects from migraine: Development and psychometric testing of the MSQOL. Headache 36:484-492

30. Martin BC, Pathak DS, Sharfman MI, Adelman JU, Taylor F, Kwong WJ, Jhingran P (2000) Validity and reliability of the Migraine-Specific Quality of Life Questionnaire (MSQ Version 2.1). Headache 40:204-215

31. Pathak DS, Chisolm DJ, Weis KA (2005) Functional Assessment in Migraine (FAIM) questionnaire: Development of an instrument based upon the WHO's International Classification of Functioning, Disability, and Health. Value Health 8:591-600

32. Leonardi M, Steiner TJ, Scher Al, Lipton RB (2005) The global burden of migraine: measuring disability in headache disorders with WHO's Classification of Functioning, Disability and Health (ICF). J Headache Pain 6: 429-440

33. Steiner TJ, Stovner $\sqcup$ (2019) Headache, functional impact and environment. Ch 11. In: Steiner TJ, Stovner $\amalg$ (eds) Societal impact of headache. Burden, costs and response. Springer Nature, Cham, pp 143-156

34. Headache Classification Committee of the International Headache Society (2018) The International Classification of Headache Disorders, 3rd edition. Cephalalgia 38: 1-211

35. International Headache Society Clinical Trials Subcommittee: Tfelt-Hansen P, Pascual J, Ramadan N, Dahlöf C, D'Amico D, Diener H-C, Møller Hansen J, Lanteri-Minet M, Loder E, McCrory D, Plancade S, Schwedt T (2012) Guidelines for controlled trials of drugs in migraine: Third edition. A guide for investigators. Cephalalgia 32: 6-38

36. Bendtsen L, Bigal ME, Cerbo R, Diener HC, Holroyd K, Lampl C, Mitsikostas $D D$, Steiner TJ, Tfelt-Hansen $P$, on behalf of the International Headache Society Clinical Trials Subcommittee (2010) Guidelines for controlled trials of drugs in tension-type headache: Second edition. Cephalalgia 30:1-16

37. Boureau F, Joubert JM, Lasserre V, Prum B, Delecoeuillerie G (1994) Doubleblind comparison of an acetaminophen $400 \mathrm{mg}$-codeine $25 \mathrm{mg}$ combination versus aspirin $1000 \mathrm{mg}$ and placebo in acute migraine attack. Cephalalgia 14:156-161

38. Diener HC, Bussone $\mathrm{G}$, de Liano H, Eikermann A, Englert R, Floeter T, Gallai V, Göbel H, Hartung E, Jimenez MD, Lange R, Manzoni GC, Mueller-Schwefe G, Nappi G, Pinessi L, Prat J, Puca FM, Titus F, Voelker M (2004) Placebocontrolled comparison of effervescent acetylsalicylic acid, sumatriptan and ibuprofen in the treatment of migraine attacks. Cephalalgia 24:947-954

39. Diener HC, Eikermann A, Gessner U, Göbel H, Haag G, Lange R, May A, Müller-Schwefe G, Voelker M (2004) Efficacy of 1,000 mg effervescent acetylsalicylic acid and sumatriptan in treating associated migraine symptoms. Eur Neurol 52:50-56

40. Diener HC, Pfaffenrath V, Pageler L, Peil H, Aicher B (2005) The fixed combination of acetylsalicylic acid, paracetamol and caffeine is more effective than single substances and dual combination for the treatment of headache: a multicentre, randomized, double-blind, single-dose, placebocontrolled parallel group study. Cephalalgia 25:776-787

41. Lange R, Schwarz JA, Hohn M (2000) Acetylsalicylic acid effervescent $1000 \mathrm{mg}$ (Aspirin) in acute migraine attacks; a multicentre, randomized, double-blind, single-dose, placebo-controlled parallel group study. Cephalalgia 20:663-667

42. Lipton RB, Goldstein J, Baggish JS, Yataco AR, Sorrentino JV, Quiring JN (2005) Aspirin is efficacious for the treatment of acute migraine. Headache 45:283-292

43. MacGregor EA, Dowson A, Davies PT (2002) Mouth-dispersible aspirin in the treatment of migraine: a placebo-controlled study. Headache 42:249-255

44. Martinez-Martin P, Raffaelli E Jr, Titus F (2001) Efficacy and safety of metamizol versus acetylsalicylic acid in patients with moderate episodic tension-type headache: a randomized, double-blind, placebo- and activecontrolled, multicentre study. Cephalalgia 21:604-610

45. Nebe J, Heier M, Diener HC (1995) Low-dose ibuprofen in self-medication of mild to moderate headache: a comparison with acetylsalicylic acid and placebo. Cephalalgia 15:531-535

46. Steiner TJ, Lange R, Voelker M (2003) Aspirin in episodic tension-type headache: placebo-controlled dose-ranging comparison with paracetamol. Cephalalgia 23:59-66

47. Steiner TJ (2015) What efficacy measures are clinically relevant and should be used in Cochrane reviews of acute migraine trials? An alternative viewpoint. Cephalalgia 35:460-462
48. Vos T, Abajobir AA, Abate KH, Abbafati C, Abbas KM, Abd-Allah F, Abdulkader RS, Abdulle AM, Abebo TA, Abera SF, Aboyans V, Abu-Raddad LJ, Ackerman IN, Adamu AA, Adetokunboh O, Afarideh M, Afshin A, Agarwal SK, Aggarwal R, Agrawal A, Agrawal S, Ahmadieh H, Ahmed MB, Aichour MTE, Aichour AN, Aichour I, Aiyar S, Akinyemi RO, Akseer N, Al Lami FH, Alahdab F, Al-Aly Z, Alam K, Alam N, Alam T et al (2017) Global, regional, and national incidence, prevalence, and years lived with disability for 328 diseases and injuries for 195 countries, 1990-2016: a systematic analysis for the Global Burden of Disease Study 2016. Lancet 390:1211-1259

49. Stovner L, Nichols E, Steiner TJ, Abd-Allah F, Abdelalim A, Al-Raddadi RM, Ansha MG, Barac A, Bensenor IM, Doan LP, Edessa D, Endres M, Foreman KJ, Gankpe FG, Gururaj G, Goulart AC, Gupta R, Hankey GJ, Hay SI, Hegazy MI, Hilawe EH, Kasaeian A, Kassa DH, Khalil I, Khang Y-H, Khubchandani J, Kim YJ, Kokubo Y, Mohammed MA, Moradi-Lakeh M, Nguyen HLT, Nirayo YL, Qorbani M, Ranta A, Roba KT et al (2018) Global, regional, and national burden of migraine and tension-type headache, 1990-2016: a systematic analysis for the Global Burden of Disease Study 2016. Lancet Neurol 17:954-976

50. GBD 2017 Disease and Injury Incidence and Prevalence Collaborators (2018) Global, regional, and national incidence, prevalence, and years lived with disability for 354 diseases and injuries for 195 countries and territories, 1990-2017: a systematic analysis for the Global Burden of Disease Study 2017. Lancet 392:1789-1858

51. GBD 2019 Diseases and Injuries Collaborators (2020) Global burden of 369 diseases and injuries in 204 countries and territories, 1990-2019: a systematic analysis for the Global Burden of Disease Study 2019. Lancet 396:1204-1222

52. Stovner L, Hagen K, Jensen R, Katsarava Z, Lipton R, Scher A, Steiner T, Zwart JA (2007) The global burden of headache: a documentation of headache prevalence and disability worldwide. Cephalalgia 27:193-210

53. Steiner TJ, Gururaj G, Andrée C, Katsarava Z, Ayzenberg I, Yu SY, Al Jumah M, Tekle-Haimanot R, Birbeck GL, Herekar A, Linde M, Mbewe E, Manandhar K, Risal A, Jensen R, Queiroz LP, Scher Al, Wang SJ, Stovner L (2014) Diagnosis, prevalence estimation and burden measurement in population surveys of headache: presenting the HARDSHIP questionnaire. J Headache Pain 15:3

54. Stovner LJ, Al Jumah M, Birbeck GL, Gururaj G, Jensen R, Katsarava Z, Queiroz LP, Scher Al, Tekle-Haimanot R, Wang SJ, Steiner TJ (2014) The methodology of population surveys of headache prevalence, burden and cost: Principles and recommendations from the Global Campaign against Headache. J Headache Pain 15:5

55. Linde M, Gustavsson A, Stovner L, Steiner TJ, Barré J, Katsarava Z, Lainez JM, Lampl C, Lantéri-Minet M, Rastenyte D, Ruiz de la Torre E, Tassorelli C, Andrée C (2012) The cost of headache disorders in Europe: the Eurolight project. Eur J Neurol 19:703-711

56. Kothari SF, Jensen RH, Steiner TJ (2021) The relationship between headache-attributed disability and lost productivity. 1. A review of the literature. J Headache Pain 22 (in press)

57. Thomas H, Kothari SF, Husøy A, Jensen RH, Katsarava Z, Tinelli M, Steiner TJ (2021) The relationship between headache-attributed disability and lost productivity. 2. Empirical evidence from population-based studies in six disparate countries. J Headache Pain 22 (in press)

58. Edmeads J, Mackell JA (2002) The economic impact of migraine: an analysis of direct and indirect costs. Headache 42:501-509

59. Selekler MH, Gökmen G, Steiner TJ (2013) Productivity impact of headache on a heavy-manufacturing workforce in Turkey. J Headache Pain 14:88

60. Salomon JA, Haagsma JA, Davis A, de Noordhout CM, Polinder S, Havelaar AH, Cassini A, Devleesschauwer B, Kretzschmar M, Speybroeck N, Murray CJ, Vos T (2015) Disability weights for the Global Burden of Disease 2013 study. Lancet Glob Health 3:e712-e723

61. Derry CJ, Derry S, Moore RA (2012) Sumatriptan (oral route of administration) for acute migraine attacks in adults. Cochrane Database Syst Rev 2:CD008615. doi:https://doi.org/10.1002/14651858.CD008615.pub2

62. Ferrari MD, Goadsby PJ, Roon KI, Lipton RB (2002) Triptans (serotonin, 5HT1B/1D agonists) in migraine: detailed results and methods of a metaanalysis of 53 trials. Cephalalgia 22:633-658

63. Tfelt-Hansen PC (2015) What efficacy measures are clinically relevant and should be used in Cochrane Reviews of acute migraine trials? A comment. Cephalalgia 35:457-459

64. Grosse SD, Lollar DJ, Campbell VA, Chamie M (2009) Disability and disabilityadjusted life years: not the same. Pub Health Rep 124:197-202

\section{Publisher's Note}

Springer Nature remains neutral with regard to jurisdictional claims in published maps and institutional affiliations. 